\title{
IncRNA/circRNA-miRNA-mRNA ceRNA network in lumbar intervertebral disc degeneration
}

\author{
JINWEN ZHU* , XINLIANG ZHANG* , WENJIE GAO, HUIMIN HU, XIAODONG WANG and DINGJUN HAO \\ Department of Spine Surgery, Honghui Hospital, Xi'an Jiaotong University, Xi'an, Shaanxi 710054, P.R. China
}

Received August 6, 2018; Accepted February 28, 2019

DOI: $10.3892 / \mathrm{mmr} .2019 .10569$

\begin{abstract}
Accumulating evidence has indicated that noncoding RNAs are involved in intervertebral disc degeneration (IDD); however, the competing endogenous RNA (ceRNA)-mediated regulatory mechanisms in IDD remain rarely reported. The present study aimed to comprehensively investigate the alterations in expression levels of circular RNA (circRNA), long noncoding RNA (lncRNA), microRNA (miRNA/miR) and mRNA in the nucleus pulposus (NP) of patients with IDD. In addition, crucial lncRNA/circRNA-miRNA-mRNA ceRNA interaction axes were screened using the GSE67567 microarray dataset obtained from the Gene Expression Omnibus database. After data preprocessing, differentially expressed circRNAs (DECs), lncRNAs (DELs), miRNAs (DEMs) or genes (DEGs) between IDD and normal controls were identified using the Linear Models for Microarray data method. A protein-protein interaction (PPI) network was constructed for DEGs based on protein databases, followed by module analysis. The ceRNA network was constructed based on the interaction between miRNAs and mRNAs, and lncRNAs/circRNAs and miRNAs. The underlying functions of mRNAs were predicted using the Database for Annotation, Visualization and Integrated Discovery database. The present study identified 636 DECs, 115 DELs, 84 DEMs and 1,040 DEGs between patients with IDD and control individuals. PPI network analysis demonstrated that Fos proto-oncogene, AP-1 transcription factor subunit (FOS), mitogen-activated protein kinase 1 (MAPK1), hypoxia inducible factor 1 subunit $\alpha$ (HIF1A) and transforming growth factor $\beta 1$ (TGFB1) were hub genes and enriched in modules. Metastasis-associated lung adenocarcinoma transcript 1 (MALAT1)/hsa_circRNA_102348-hsa-miR-185-5p-TGFB1/FOS,
\end{abstract}

Correspondence to: Dr Dingjun Hao, Department of Spine Surgery, Honghui Hospital, Xi'an Jiaotong University, 555 Youyi East Road, Xi'an, Shaanxi 710054, P.R. China

E-mail: 3058867162@qq.com

${ }^{*}$ Contributed equally

Key words: intervertebral disc degeneration, nucleus pulposus, competing endogenous RNA, long noncoding RNA, microRNA, circular RNA
MALAT1-hsa-miR-155-5p-HIF1A, hsa_circRNA_102399-hs a-miR-302a-3p-HIF1A, MALAT1-hsa-miR-519d-3p-MAPK1 and hsa_circRNA_100086-hsa-miR-509-3p-MAPK1 ceRNA axes were obtained by constructing the ceRNA networks. In conclusion, these identified ceRNA interaction axes may be crucial targets for the treatment of IDD.

\section{Introduction}

Intervertebral disc degeneration (IDD) is characterized by excessive apoptosis of nucleus pulposus (NP) cells and degradation of extracellular matrix (ECM) components, and is considered the main contributing factor to lower back pain (LBP). It is estimated that $\sim 84 \%$ of people will experience LBP during their lives worldwide, with $10 \%$ becoming chronically disabled (1), thus seriously affecting quality of life and imposing heavy economic burdens on families and society. Currently, clinical interventions for IDD primarily include conservative medication and surgery (spinal fusion or total disc replacement); however, these treatments are only able to temporarily relieve pain symptoms, without solving the underlying issues in IDD and providing a permanent cure $(2,3)$. Therefore, it is necessary to deeply investigate the underlying mechanisms of IDD, in order to develop more effective strategies for preventing and treating IDD-associated LBP.

Recently, emerging evidence has suggested that noncoding RNAs, including microRNAs (miRNAs/miRs), long noncoding RNAs (lncRNAs) and circular RNAs (circRNAs) serve crucial roles in various biological processes, including cell proliferation and apoptosis (4). miRNAs function by binding to complementary sequences in the 3'-untranslated region (UTR) of their target mRNAs, thereby triggering either translational inhibition or mRNA degradation of the transcript (5). lncRNAs/circRNAs may act as competing endogenous RNAs (ceRNAs) by competitively binding to miRNAs through their miRNA response elements, thus regulating the expression levels of miRNA target mRNAs (6). Therefore, lncRNA/circRNA-miRNA-mRNA interactions may be an important mechanism underlying the initiation and development of IDD. This hypothesis has been verified in previous studies. Notably, Xi et al (7) demonstrated that lncRNA HLA complex group 18 (HCG18) is significantly upregulated in patients with IDD and its expression is positively correlated with disc degeneration grade. Subsequently, a luciferase reporter assay was conducted, which indicated 
that HCG18 may act as an endogenous sponge to downregulate miR-146a-5p expression in NP cells, thus promoting the upregulation of a miR-146a-5p target gene, TNF-receptor associated factor 6, ultimately suppressing the growth of NP cells by decreasing cell numbers in $\mathrm{S}$ phase of the cell cycle, inducing cell apoptosis, recruiting macrophages and hypercalcification (7). Wang et al (8) demonstrated that lncRNA RP11-296A18.3 interacts with miR-138 to induce upregulation of the miR-138 target gene, hypoxia inducible factor 1 subunit $\alpha$ (HIF1A), thus affecting NP proliferation and ECM synthesis. The expression of RP11-296A18.3 is positively correlated with HIF1A; however, RP11-296A18.3 and HIF1A are inversely correlated with miR-138 in IDD tissues (8). In addition, by circRNA microarray assay, bioinformatics analysis, RNA immunoprecipitation and luciferase assay, Wang et al (9) provided evidence to suggest that circRNA-4099 is able to function as a 'sponge' by competitively binding miR-616-5p, which reverses the suppression of SRY-box 9 by miR-616-5p. Cheng et al (10) revealed that circVMA21 acts as a sponge of miR-200c, thus regulating the expression of the target mRNA, $\mathrm{X}$-linked inhibitor of apoptosis (XIAP). The decreased expression of XIAP in inflammatory cytokines-treated NP cells and degenerative NP tissues is directly associated with excessive apoptosis, and an imbalance between the anabolic and catabolic factors of ECM. Conversely, intradiscal injection of circVMA21 may alleviate IDD in a rat model (10). These findings indicated that targeting the regulatory effects of associated lncRNAs, circRNAs, miRNAs and mRNAs may have a potential role in the clinical treatment of IDD. However, to the best of our knowledge, IDD-associated lncRNA/circRNA-miRNA-mRNA ceRNA regulatory mechanisms remain rarely reported, until now (11).

The present study aimed to preliminarily identify novel lncRNA/circRNA-miRNA-mRNA ceRNA-mediated regulatory mechanisms in IDD by constructing a ceRNA regulatory network using microarray data collected from a public database. This study may provide targets for the development of novel therapeutic strategies to treat IDD.

\section{Materials and methods}

Gene expression omnibus (GEO) dataset collection. A microarray dataset was retrieved from the public GEO database (www.ncbi.nlm.nih.gov/geo); the accession number of the dataset used is GSE67567, which contains three sub-datasets: GSE67566, circRNA expression profile (platform: GPL19978, Agilent-069978 Arraystar Human CircRNA microarray V1; Agilent Technologies, Inc., Santa Clara, CA, USA) $(12,13)$; GSE63492, miRNA expression profile [platform: GPL19449, Exiqon miRCURY LNA microRNA Array, 7th generation REV-hsa, mmu \& rno (miRBase v18.0); Exiqon; Qiagen, Inc., Valencia, CA, USA] $(12,13)$; and GSE56081, mRNA-IncRNA expression profile [platform: GPL15314, Arraystar Human LncRNA microarray V2.0 (Agilent_033010 Probe Name version); Agilent Technologies, Inc.] $(12,14)$. These three sub-datasets included NP samples derived from five normal control individuals and five patients with IDD.

Data preprocessing and differential expression analysis. The raw TXT data collected from the microarray platforms were preprocessed using the Linear Models for Microarray data (LIMMA) method (15) (version 3.34.0; www.bioconductor.org/packages/release/bioc/html/limma.html) in the Bioconductor R package (version 3.4.1; www.R-project.org), including base-2 logarithmic ( $\log 2)$ transformation and quantile normalization. For the GSE56081 microarray data, the probe sequences were downloaded from the annotation platforms and aligned with the human genome using Clustal W computer program (version 2; www.clustal.org) to obtain the expression levels of lncRNA and mRNAs.

The lists of differentially expressed genes (DEGs), differentially expressed lncRNAs (DELs), differentially expressed circRNAs (DECs) and differentially expressed miRNAs (DEMs) between controls and patients with IDD were generated using the LIMMA method (15), where statistical significance was set as $\mid \log$-fold change $(\log F C) \mid>1$ and Benjamini and Hochberg-corrected (16) false discovery rates (FDR)<0.05. A hierarchical cluster heatmap representing expression intensity and direction was created using pheatmap in $\mathrm{R}$ package (version: 1.0.8; cran.r-project.org/web/packages/pheatmap) based on Euclidean distance.

Protein-protein interaction (PPI) network. The PPI network of DEGs was constructed and visualized using Cytoscape software (version 3.6.1; www.cytoscape.org) (17) based on the interaction data from the Search Tool for the Retrieval of Interacting Genes (version 10.0; string db.org) database (18). The topological features of the PPI network, including degree [the number of edges (interactions) of a node (protein)], betweenness centrality (BC, the number of shortest paths that run through a node), closeness centrality ( $\mathrm{CC}$, the average length of the shortest paths to access all other proteins in the network) and average path length, were calculated, in order to determine the crucial genes using the CytoNCA plugin in Cytoscape software (apps.cytoscape.org/apps/cytonca) (19).

The Molecular Complex Detection(MCODE; version:1.4.2; apps.cytoscape.org/apps/mcode) (20) plugin in Cytoscape software was used to identify functionally related and highly interconnected modules from the PPI network with a degree cutoff of 2 , node score cutoff of 0.2 , k-core of 2 and maximum depth of 100 .

miRNA regulatory network construction. The DEM-associated target genes were predicted using the starBase database (version 2.0; starbase.sysu.edu.cn/index.php) (21) which provides the prediction results of five miRNA databases (TargetScan, picTar, RNA22, PITA and miRanda). The miRNA-target gene interaction pairs were selected if they were predicted in $\geq 1$ database. The target genes were then overlapped with the DEGs, and the negative interaction pairs between DEMs and DEGs (according to their expression levels) were used to construct the miRNA-mRNA network using Cytoscape software (version 3.6.1; www.cytoscape.org) (17). The known miRNAs associated with IDD were predicted using the Human microRNA Disease Database (HMDD; www.cuilab. cn/hmdd) (22).

CeRNA regulatory network construction. The starBase database (version 2.0; starbase.sysu.edu.cn/index.php) (21) database was used to screen the interactions between DELs and DEMs, 
which were then integrated with the miRNA-mRNA interactions to establish the DEL-DEM-DEG ceRNA network using Cytoscape software (version 3.6.1; www.cytoscape.org) (17).

Human sequences of DEMs and DECs were downloaded from the circBase (www.circbase.org) (23) and miRBase (version 21; www.mirbase.org) (24) databases, respectively. miRanda (cbio.mskcc.org/miRNA2003/miranda.html) (25) was used to predict the interactions between DECs and DEMs according to the following parameter settings: Gap Open Penalty, -8; Gap Extend, -2; Score Threshold, 80\%; and Energy Threshold, -20. The interaction pairs between DECs and DEMs were then integrated with the miRNA-mRNA interactions to establish the DEC-DEM-DEG ceRNA network using Cytoscape software (version 3.6.1; www.cytoscape.org) (17).

The overlapped miRNA-mRNAs in the above two ceRNA networks were also selected to construct the lncRNA/circRNA-miRNA-mRNA network.

Functional enrichment analysis. The Kyoto Encyclopedia of Genes and Genomes (KEGG) pathway enrichment analysis of genes in each module and network was conducted using the Database for Annotation, Visualization and Integrated Discovery (DAVID) online tool (version 6.8; http://david.abcc. ncifcrf.gov) (26), with $\mathrm{P}<0.05$ set as the cut-off value. In addition, all known IDD-associated pathways were downloaded from the Comparative Toxicogenomics Database (CTD; ctd. mdibl.org) (27), which were then overlapped with the enriched ceRNA pathways, in order to obtain an IDD pathway-associated ceRNA network.

\section{Results}

Differential expression analysis. According to the pre-set threshold $(\mathrm{FDR}<0.05$ and $\mid \log \mathrm{FCl}>1)$, a total of $636 \mathrm{DECs}$ were identified between IDD and control samples, including 354 upregulated and 282 downregulated circRNAs; 115 DELs were screened, consisting of 50 upregulated and 65 downregulated lncRNAs; 84 DEMs were obtained, containing 50 upregulated and 34 downregulated miRNAs; and 1,040 DEGs were generated, comprising 763 upregulated and 277 downregulated genes. The top 20 DECs, DELs, DEMs and DEGs are presented in Table I. The hierarchical cluster heat maps indicated that these DECs (Fig. 1A), DELs (Fig. 1B), DEMs (Fig. 1C) and DEGs (Fig. 1D) could distinguish IDD from control samples.

PPI network. A PPI network was constructed using the screened DEGs, which included 721 nodes (561 upregulated and 160 downregulated) and 3,561 interaction pairs. DNA topoisomerase II $\beta$, matrix metallopeptidase 2 (MMP2), enolase (ENO)1, Fos proto-oncogene, AP-1 transcription factor subunit (FOS), mitogen-activated protein kinase 1 (MAPK1), HIF1A, protein phosphatase 2 scaffold subunit $\mathrm{A} \alpha$ (PPP2R1A), ENO2, RNA polymerase II subunit C (POLR2C), transforming growth factor $\beta 1$ (TGFB1), fibronectin 1 (FN1), Jun proto-oncogene, AP-1 transcription factor subunit (JUN), vimentin and $\mathrm{H} 2 \mathrm{~A}$ histone family member V (H2AFV) were considered hub genes in the PPI network because they were present in the top 35 genes of four topological features (Tables II and III). Six functionally related and highly interconnected modules were subsequently extracted using MCODE, in order to further screen crucial genes (Fig. 2). Among them, the hub genes PPP2R1A, TGFB1, POLR2C and FN1 were included in module 2; H2AFV was included in module 4; FOS was included in module 5; and MMP2 and MAPK1 were contained in module 6 .

The genes in each module were then subjected to analysis using DAVID, in order to predict their functions. The results of KEGG enrichment analysis (Table IV) demonstrated that in module 2, PPP2R1A was enriched in 'mRNA surveillance pathway' and TGFB1 was enriched in 'Cytokine-cytokine receptor interaction'; in module 4, H2AFV was enriched in 'Systemic lupus erythematosus'; in module 5, FOS was involved in 'Rheumatoid arthritis' and 'Human T-cell leukemia virus 1 infection'; and in module 6, MAPK1 and MMP2 participated in 'Estrogen signaling pathway'. The other hub genes were not enriched in KEGG pathways.

miRNA regulatory network. A total of 305 differentially expressed target genes were predicted for 14 of the 84 DEMs, which were used to construct the miRNA-mRNA network (Fig. 3). This network included 522 negative interaction relationships between 14 DEMs and 305 DEGs (six upregulated DEMs regulated 45 downregulated DEGs; eight downregulated DEMs regulated 260 upregulated DEGs, such as hsa-miR-155-5p/hsa-miR-302a-3p/hsa-miR-519d-3p-HIF1A, hsa-miR-185-5p-TGFB1,hsa-miR-185-5p/hsa-miR-155-5p-FOS and hsa-miR-509-3p/hsa-miR-519d-3p-MAPK1). Among all of the DEMs in this miRNA regulatory network, hsa-miR-155-5p was revealed to be associated with IDD, as determined by searching the HMDD database.

The underlying functions of the DEGs in the miRNA-mRNA network were also analyzed by DAVID database. The results indicated that 'TGF-beta signaling pathway' (MAPK1 and TGFB1), 'Adherens junction' (MAPK1), 'MAPK signaling pathway' (MAPK1, TGFB1 and FOS), 'Cell cycle' (TGFB1), 'mTOR signaling pathway' (MAPK1 and HIF1A), 'Toll-like receptor signaling pathway' (MAPK1 and FOS), 'B cell receptor signaling pathway' (MAPK1 and FOS) and 'T cell receptor signaling pathway' (MAPK1 and FOS) pathways were significantly enriched for genes in the miRNA-mRNA network (Table V).

CeRNA network. Using the starBase database, nine DEMs were predicted to regulate 12 DELs; this was used to establish the IncRNA-miRNA-mRNA ceRNA network via integration with the miRNA-mRNA network (Fig. 4). This network comprised 280 nodes (nine DEMs; 12 DELs; 259 DEGs) and 407 interactions (16 DEL-DEM and 391 DEM-DEG interactions). Notably, upregulated metastasis-associated lung adenocarcinoma transcript 1 (MALAT1) may function as a ceRNA to suppress the inhibitory effects of hsa-miR-155-5p on HIF1A and FOS, thus leading to their upregulated expression. Similarly, upregulated MALAT1 may regulate the targeted effects of hsa-miR-185-5p on TGFB1 and FOS, as well as hsa-miR-519d-3p on HIF1A and MAPK1. The functional analysis of genes in the IncRNA-associated ceRNA network were significantly enriched in 'TGF-beta signaling pathway', 'FoxO signaling pathway' and 'TNF signaling pathway' (Table V); 
Table I. Top upregulated and downregulated differentially expressed circRNAs, IncRNAs, miRNAs and mRNAs identified from Gene Expression Omnibus microarray datasets.

\begin{tabular}{lcc}
\hline A, circRNAs & & \\
\hline RNA & logFC & FDR \\
\hline hsa_circRNA_101852 & 2.98 & $3.92 \times 10^{-15}$ \\
hsa_circRNA_101853 & 2.93 & $6.98 \times 10^{-16}$ \\
hsa_circRNA_101139 & 2.92 & $6.98 \times 10^{-16}$ \\
hsa_circRNA_103890 & 2.86 & $1.72 \times 10^{-15}$ \\
hsa_circRNA_400019 & 2.84 & $3.87 \times 10^{-14}$ \\
hsa_circRNA_102324 & 2.78 & $1.00 \times 10^{-15}$ \\
hsa_circRNA_104703 & 2.72 & $1.24 \times 10^{-15}$ \\
hsa_circRNA_104600 & 2.68 & $7.51 \times 10^{-15}$ \\
hsa_circRNA_100604 & 2.68 & $1.57 \times 10^{-15}$ \\
hsa_circRNA_100018 & 2.61 & $1.67 \times 10^{-15}$ \\
hsa_circRNA_103410 & 2.59 & $6.98 \times 10^{-16}$ \\
hsa_circRNA_000200 & 2.56 & $2.32 \times 10^{-14}$ \\
hsa_circRNA_100086 & 2.32 & $7.03 \times 10^{-14}$ \\
hsa_circRNA_102348 & 1.95 & $9.57 \times 10^{-14}$ \\
hsa_circRNA_102399 & 1.63 & $4.92 \times 10^{-12}$ \\
hsa_circRNA_101645 & -3.30 & $1.60 \times 10^{-14}$ \\
hsa_circRNA_104508 & -3.26 & $2.19 \times 10^{-13}$ \\
hsa_circRNA_102116 & -3.18 & $1.92 \times 10^{-14}$ \\
hsa_circRNA_103838 & -3.06 & $6.98 \times 10^{-16}$ \\
hsa_circRNA_101557 & -3.05 & $1.96 \times 10^{-14}$ \\
\hline
\end{tabular}

$\mathrm{B}, \operatorname{lncRNAs}$

\begin{tabular}{lcc}
\hline RNA & $\log$ FC & FDR \\
\hline TRPC7-AS1 & 6.61 & $6.55 \times 10^{-8}$ \\
MIR4458HG & 1.40 & $5.56 \times 10^{-3}$ \\
GAS5 & 1.40 & $4.30 \times 10^{-2}$ \\
CBR3-AS1 & 1.40 & $3.05 \times 10^{-4}$ \\
ADPGK-AS1 & 1.40 & $2.67 \times 10^{-3}$ \\
SNHG5 & 1.40 & $2.67 \times 10^{-2}$ \\
ADARB2-AS1 & 1.391 & $4.17 \times 10^{-3}$ \\
LINC00431 & 1.39 & $4.68 \times 10^{-4}$ \\
MCCC1-AS1 & 1.39 & $8.89 \times 10^{-3}$ \\
MALAT1 & 1.07 & $6.58 \times 10^{-4}$ \\
HOTAIR & -7.21 & $6.47 \times 10^{-8}$ \\
LINC00957 & -6.28 & $7.33 \times 10^{-9}$ \\
VPS13A-AS1 & -6.01 & $1.81 \times 10^{-8}$ \\
IL10RB-AS1 & -5.54 & $9.48 \times 10^{-9}$ \\
MAPT-AS1 & -5.12 & $6.53 \times 10^{-9}$ \\
LINC00689 & -3.63 & $2.87 \times 10^{-5}$ \\
EFCAB6-AS1 & -3.39 & $2.96 \times 10^{-8}$ \\
HAND2-AS1 & -3.21 & $3.47 \times 10^{-4}$ \\
LINC00884 & -3.12 & $5.11 \times 10^{-5}$ \\
LINC01405 & -3.04 & $9.52 \times 10^{-4}$ \\
\hline
\end{tabular}

C, miRNAs

\begin{tabular}{lcc}
\hline RNA & $\operatorname{logFC}$ & FDR \\
\hline hsa-miR-4287 & 5.81 & $4.50 \times 10^{-3}$
\end{tabular}

Table I. Continued.

C, miRNAs

\begin{tabular}{lcc}
\hline RNA & $\operatorname{logFC}$ & FDR \\
\hline hsa-miR-3150a-3p & 5.03 & $1.24 \times 10^{-5}$ \\
hsa-miR-3157-3p & 4.90 & $1.00 \times 10^{-2}$ \\
hsa-miR-660-5p & 4.80 & $1.99 \times 10^{-34}$ \\
hsa-miR-887-3p & 4.05 & $1.05 \times 10^{-4}$ \\
hsa-miR-5010-5p & 4.03 & $2.66 \times 10^{-2}$ \\
hsa-miR-933 & 3.43 & $7.39 \times 10^{-3}$ \\
hsa-miR-3127-5p & 3.35 & $4.45 \times 10^{-3}$ \\
hsa-miR-4450 & 3.24 & $2.23 \times 10^{-3}$ \\
hsa-miR-516a-5p & 3.15 & $2.29 \times 10^{-4}$ \\
hsa-miR-1184 & -4.85 & $5.44 \times 10^{-4}$ \\
hsa-miR-125b-1-3p & -4.69 & $4.44 \times 10^{-4}$ \\
hsa-miR-486-3p & -4.39 & $8.00 \times 10^{-3}$ \\
hsa-miR-3648 & -3.76 & $6.14 \times 10^{-46}$ \\
hsa-miR-196b-5p & -3.43 & $1.00 \times 10^{-2}$ \\
hsa-miR-155-5p & -3.22 & $2.00 \times 10^{-2}$ \\
hsa-miR-302a-3p & -1.93 & $2.00 \times 10^{-2}$ \\
hsa-miR-519d-3p & -1.81 & $4.00 \times 10^{-2}$ \\
hsa-miR-509-3p & -1.78 & $3.00 \times 10^{-2}$ \\
hsa-miR-185-5p & -1.53 & $1.00 \times 10^{-2}$ \\
\hline
\end{tabular}

D, mRNAs

\begin{tabular}{llc}
\hline RNA & $\operatorname{logFC}$ & FDR \\
\hline HBB & 8.41 & $3.87 \times 10^{-9}$ \\
HBA1 & 8.19 & $8.62 \times 10^{-11}$ \\
COL1A2 & 7.01 & $3.41 \times 10^{-10}$ \\
PTP4A3 & 6.98 & $7.31 \times 10^{-7}$ \\
RBM38 & 6.70 & $1.72 \times 10^{-9}$ \\
MFAP4 & 6.19 & $7.41 \times 10^{-9}$ \\
GREM1 & 5.77 & $4.49 \times 10^{-8}$ \\
NKG7 & 5.55 & $1.13 \times 10^{-9}$ \\
TREM1 & 5.47 & $1.12 \times 10^{-9}$ \\
LUM & 5.36 & $3.28 \times 10^{-9}$ \\
PHLDB2 & -7.09 & $2.99 \times 10^{-8}$ \\
TMEM177 & -6.96 & $1.12 \times 10^{-9}$ \\
KIAA0319 & -6.95 & $5.16 \times 10^{-10}$ \\
ERLIN1 & -6.89 & $6.61 \times 10^{-10}$ \\
APOD & -6.47 & $4.61 \times 10^{-7}$ \\
SLF2 & -6.45 & $5.61 \times 10^{-10}$ \\
NDRG4 & -6.44 & $4.91 \times 10^{-10}$ \\
GUCY1A3 & -6.43 & $9.45 \times 10^{-10}$ \\
PLAGL1 & -6.41 & $1.67 \times 10^{-7}$ \\
ATP8B3 & -6.32 & $7.34 \times 10^{-9}$ \\
\hline PHi & & \\
\hline
\end{tabular}

Positive $\log \mathrm{FC}$ values indicate upregulated expression in IDD samples compared with in control samples, whereas negative $\operatorname{logFC}$ values indicate downregulated expression in IDD samples compared with in control samples. All microarray datasets were retrieved from public Gene Expression Omnibus databases, including GSE67566 $(12,13)$ for circRNA expression; GSE63492 $(12,13)$ for miRNA expression and GSE56081 $(12,14)$ for mRNA-lncRNA expression. circRNA, circular RNA; FC, fold change; FDR, false discovery rates; IDD, intervertebral disc degeneration; lncRNA, long noncoding RNA; miRNA, microRNA. 

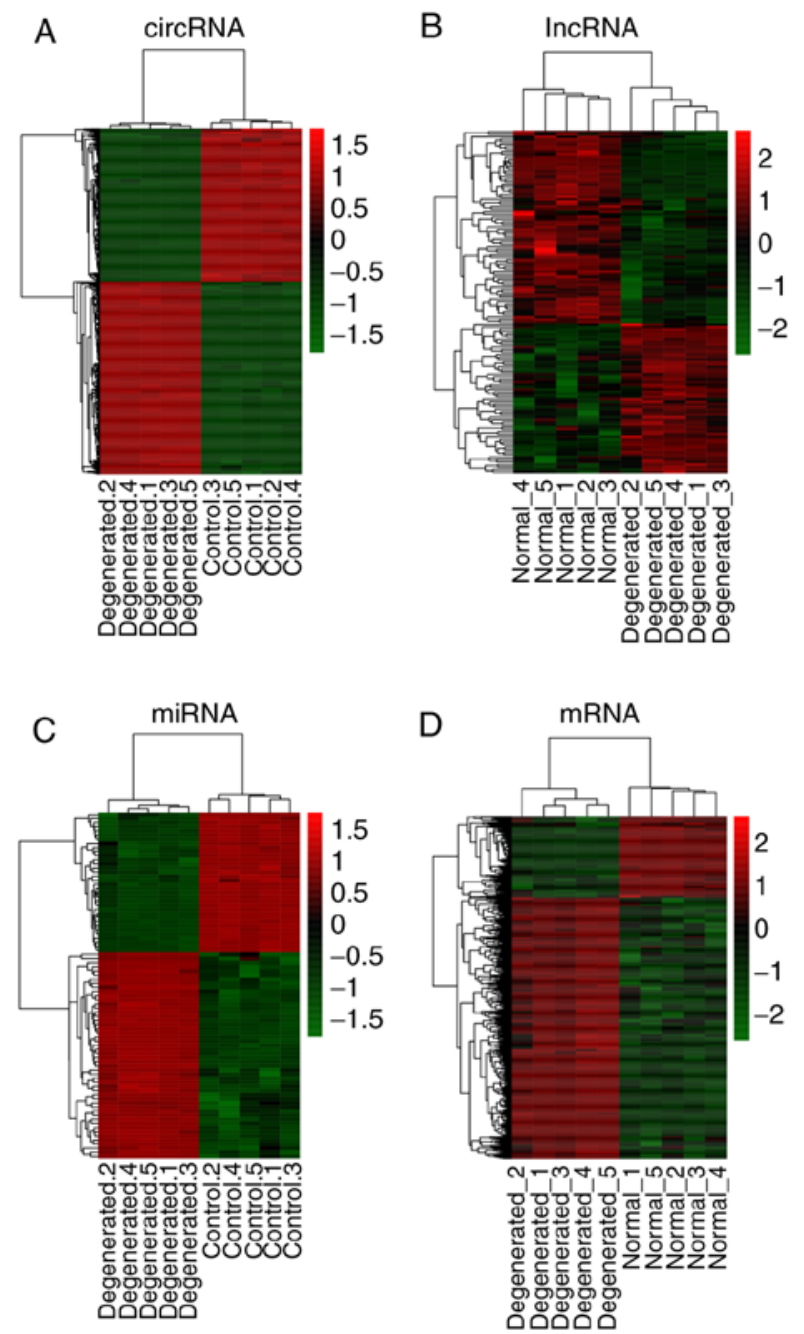

Figure 1. Hierarchical clustering and heat map analysis of differentially expressed (A) circRNAs, (B) lncRNAs, (C) miRNAs and (D) genes in intervertebral disc degeneration. circRNA, circular RNA; lncRNA, long noncoding RNA; miRNA, microRNA.

MAPK1, FOS and TGFB1 were each included in at least one of these pathways.

Using the miRanda database, 61 DEMs were predicted to regulate 63 DECs; this information was used to establish the circRNA-miRNA-mRNA ceRNA network via integration with the miRNA-mRNA network (Fig. 5). Notably, upregulated hsa_circRNA_102348 may function as a ceRNA to suppress the inhibitory effects of hsa-miR-185-5p on TGFB1 and FOS, thus resulting in their upregulated expression; upregulated hsa_circRNA_102399 may act as a ceRNA to regulate the hsa-miR-302a-3p-HIF1A interaction; hsa_circRNA_100086 may influence the regulatory effect of hsa-miR-509-3p on MAPK1. hsa_circRNA_102348 was selected because it has previously been studied in other diseases (28). In addition, hsa_circRNA_102399 (score threshold, 92\%) and hsa_circRNA_100086 (score threshold, 90\%) may be important in IDD, because they interacted with their miRNAs with the highest score thresholds and the expression trend of these circRNAs was opposite to that of their target miRNAs. Functional analysis of genes in the circRNA-related ceRNA network revealed that they were significantly enriched in 'TGF-beta signaling pathway' and 'MAPK signaling pathway';
MAPK1, TGFB1 and FOS were each included in at least one of these pathways (Table V).

Through analysis of the IncRNA and circRNA ceRNA networks, it was revealed that hsa-miR-185-5p,hsa-miR-486-5p, hsa-miR-196b-5p, hsa-miR-382-5p and hsa-miR-324-5p were includedinboth; therefore, alncRNA/circRNA-miRNA-mRNA integrated network was also established (Fig. 6), in which hsa-miR-185-5p-TGFB1-associated ceRNA axes may be particularly important because TGFB1 was enriched in most of the KEGG pathways for this network (Table V).

IDD pathway-related ceRNA network. Using 'intervertebral disc degeneration' as the key search term, 30 KEGG pathways were screened from the CTD database; four pathways were overlapped with those enriched for genes in the integrated ceRNA network, including 'TGF-beta signaling pathway', 'FoxO signaling pathway', 'Endocytosis' and 'Cell cycle'. The pathway-related genes were extracted to construct the IDD pathway-related ceRNA network (Fig. 7), in which the hsa circRNA_102348/MALAT1-hsa-miR-185-5p-TGFB1 ceRNA axes were contained.

\section{Discussion}

The present study identified FOS, MAPK1, HIF1A and TGFB1 as crucial genes in IDD, as determined by topological feature analysis of genes in a PPI network and module screening. Subsequently, by constructing a ceRNA network, it was suggested that the upregulated lncRNA MALAT1 may be particularly important for IDD, as it may function as a ceRNA for downregulating hsa-miR-155-5p, hsa-miR-185-5p and hsa-miR-519d-3p expression, thus leading to the upregulation of FOS, TGFB1, HIF1A and MAPK1, respectively. In addition, upregulated hsa_circRNA_102348, hsa_circRNA_102399 and hsa_circRNA_100086 may function as ceRNAs to block the inhibitory effects of hsa-miR-185-5p, hsa-miR-302a-3p and hsa-miR-509-3p on the expression levels of TGFB1/FOS, HIF1A and MAPK1, respectively. MAPK1 and FOS may be involved in IDD by influencing the MAPK pathway, thus affecting inflammatory pathways. KEGG analysis suggested that TGFB1 participated in IDD by affecting 'TGF-beta signaling pathway', 'Cell cycle' and 'Cytokine-cytokine interaction', whereas HIF1A may be associated with 'mTOR signaling pathway'.

It has been reported that TGFB promotes NP cell proliferation and stimulates ECM synthesis (11). Therefore, overexpression of TGFB may have the potential to inhibit IDD and exert therapeutic effects; this has been demonstrated in several in vivo studies (29,30). Further studies have indicated that TGFB exerts anabolic effects on intervertebral discs by antagonizing inflammation (31,32). Furthermore, Zhang et al (33) reported that the expression levels of TGFB1 and C-C motif chemokine ligand (CCL)3/4 are elevated in degenerative NP tissue. TGFB1 treatment significantly inhibits CCL4 expression and prevents pain development in a rat model of IDD; this effect is dependent on the extracellular signal-regulated kinase 1/2 (ERK1/2) signaling pathway. Yang et al $(34,35)$ observed that the inflammatory cytokine tumor necrosis factor (TNF)- $\alpha$ induces high expression of syndecan-4 in NP cells, which is required for MMP3 activation to trigger the progression of 
Table II. Topological features of DEGs in the protein-protein interaction network.

\begin{tabular}{|c|c|}
\hline DEG & Value \\
\hline MAPK1 & 86 \\
\hline JUN & 78 \\
\hline TOP2B & 75 \\
\hline TGFB1 & 70 \\
\hline FOS & 58 \\
\hline BTRC & 49 \\
\hline FN1 & 49 \\
\hline $\mathrm{H} 2 \mathrm{AFV}$ & 44 \\
\hline SOCS3 & 43 \\
\hline POLR2C & 43 \\
\hline UBE2C & 43 \\
\hline CDC23 & 42 \\
\hline VIM & 42 \\
\hline KEAP1 & 41 \\
\hline MMP2 & 40 \\
\hline ANAPC1 & 40 \\
\hline SKP2 & 40 \\
\hline HIST2H3A & 40 \\
\hline SMAD3 & 39 \\
\hline ENO2 & 39 \\
\hline SMAD2 & 38 \\
\hline CDC26 & 38 \\
\hline UBE2G1 & 37 \\
\hline H2AFX & 37 \\
\hline CSF2 & 37 \\
\hline ENO1 & 37 \\
\hline HIST2H2AA3 & 36 \\
\hline UBE2R2 & 36 \\
\hline HIST1H2BK & 36 \\
\hline PPP2R1A & 36 \\
\hline SIAH1 & 36 \\
\hline RPSA & 36 \\
\hline FAU & 35 \\
\hline HERC3 & 34 \\
\hline HIF1A & 33 \\
\hline
\end{tabular}

B, CC

\begin{tabular}{lc}
\hline DEG & Value \\
\hline ST6GALNAC1 & 1.000 \\
OTOP3 & 1.000 \\
ZDHHC9 & 1.000 \\
HID1 & 1.000 \\
TMEM128 & 1.000 \\
RAPH1 & 1.000 \\
LANCL1 & 1.000 \\
CAMTA1 & 1.000 \\
DPP10 & 1.000 \\
GTF2H2C & 1.000
\end{tabular}

Table II. Continued.

\begin{tabular}{|c|c|}
\hline \multicolumn{2}{|l|}{$\mathrm{B}, \mathrm{CC}$} \\
\hline DEG & Value \\
\hline BRD9 & 1.0000 \\
\hline ST6GALNAC4 & 0.6667 \\
\hline SLC25A43 & 0.6667 \\
\hline ORMDL1 & 0.6667 \\
\hline GALNT1 & 0.6667 \\
\hline JUN & 0.4257 \\
\hline MAPK1 & 0.4250 \\
\hline FOS & 0.4001 \\
\hline TGFB1 & 0.3967 \\
\hline TOP2B & 0.3917 \\
\hline VIM & 0.3842 \\
\hline FN1 & 0.3766 \\
\hline MMP2 & 0.3762 \\
\hline POLR2C & 0.3760 \\
\hline SMAD3 & 0.3752 \\
\hline YBX1 & 0.3748 \\
\hline ENO1 & 0.3726 \\
\hline HIF1A & 0.3726 \\
\hline ACTL6A & 0.3720 \\
\hline HBA1 & 0.3714 \\
\hline ENO2 & 0.3707 \\
\hline $\mathrm{H} 2 \mathrm{AFV}$ & 0.3674 \\
\hline $\mathrm{H} 2 \mathrm{AFX}$ & 0.3674 \\
\hline SMAD2 & 0.3668 \\
\hline PPP2R1A & 0.3651 \\
\hline \multicolumn{2}{|l|}{$\mathrm{C}, \mathrm{BC}$} \\
\hline DEG & Value \\
\hline ST6GALNAC1 & 1.0000 \\
\hline ZDHHC9 & 1.0000 \\
\hline MAPK1 & 0.1255 \\
\hline TOP2B & 0.0988 \\
\hline JUN & 0.0913 \\
\hline TGFB1 & 0.0619 \\
\hline FOS & 0.0450 \\
\hline NME2 & 0.0413 \\
\hline FN1 & 0.0365 \\
\hline PPP2R1A & 0.0319 \\
\hline VIM & 0.0314 \\
\hline POLR2C & 0.0310 \\
\hline RAD51 & 0.0301 \\
\hline HIF1A & 0.0293 \\
\hline CTSD & 0.0272 \\
\hline ENO2 & 0.0266 \\
\hline ACTG2 & 0.0253 \\
\hline ENO1 & 0.0242 \\
\hline NDUFA4 & 0.0239 \\
\hline H2AFV & 0.0227 \\
\hline MMP2 & 0.0224 \\
\hline
\end{tabular}


Table II. Continued.

\begin{tabular}{lc}
\hline C, BC & \\
\hline DEG & Value \\
\hline SNCA & 0.0209 \\
SOCS3 & 0.0205 \\
CSF2 & 0.0201 \\
HBA1 & 0.0200 \\
DCN & 0.0199 \\
HLA-DRB1 & 0.0195 \\
BTRC & 0.0182 \\
TGFBR2 & 0.0182 \\
DICER1 & 0.0182 \\
COL1A2 & 0.0181 \\
HIST2H3A & 0.0180 \\
ATF4 & 0.0180 \\
ACTL6A & 0.0170 \\
GNG4 & 0.0166
\end{tabular}

\section{D, APL}

\begin{tabular}{lc}
\hline DEG & Value \\
\hline
\end{tabular}

\section{ST6GALNAC1}

ZDHHC9

OTOP3

HID1

TMEM128

RAPH1

LANCL1

CAMTA1

DPP10

GTF2H2C

BRD9

ST6GALNAC4

SLC25A43

ORMDL1

1.0000

1.0000

1.0000

1.0000

1.0000

1.0000

1.0000

1.0000

1.0000

1.0000

1.0000

1.5000

1.5000

1.5000

GALNT1

JUN

1.5000

2.3489

MAPK1

2.3532

FOS

TGFB1

TOP2B

VIM

FN1

MMP2

POLR2C

SMAD3

YBX1

HIF1A

ENO1

ACTL6A

HBA1

ENO2
Table II. Continued.

\begin{tabular}{lc}
$\mathrm{D}, \mathrm{APL}$ & \\
\hline $\mathrm{DEG}$ & Value \\
\hline H2AFV & 2.7220 \\
H2AFX & 2.7220 \\
SMAD2 & 2.7262 \\
PPP2R1A & 2.7390
\end{tabular}

Value refers to degree, CC, BC or APL, accordingly. APL, average path length; $\mathrm{BC}$, betweenness centrality; $\mathrm{CC}$, closeness centrality; DEGs, differentially expressed genes.

Table III. Overlapping DEGs according to topological features (degree, closeness centrality, betweenness centrality and average path length).

\begin{tabular}{lcc}
\hline DEG & $\log$ FC & \multicolumn{1}{c}{ FDR } \\
\hline TOP2B & -2.97 & $1.72 \times 10^{-6}$ \\
MMP2 & -2.56 & $1.58 \times 10^{-3}$ \\
ENO1 & 2.43 & $1.3 \times 10^{-6}$ \\
FOS & 2.32 & $2.18 \times 10^{-6}$ \\
MAPK1 & 2.38 & $1.45 \times 10^{-3}$ \\
HIF1A & 2.97 & $4.53 \times 10^{-4}$ \\
PPP2R1A & 2.15 & $2.3 \times 10^{-7}$ \\
ENO2 & 3.37 & $1.04 \times 10^{-5}$ \\
POLR2C & 3.01 & $2.00 \times 10^{-7}$ \\
TGFB1 & 2.75 & $3.80 \times 10^{-5}$ \\
FN1 & 4.69 & $2.90 \times 10^{-5}$ \\
JUN & 2.22 & $1.05 \times 10^{-6}$ \\
VIM & 5.26 & $1.13 \times 10^{-9}$ \\
H2AFV & 2.16 & $3.41 \times 10^{-4}$ \\
\hline
\end{tabular}

Positive $\log \mathrm{FC}$ values refer to upregulated expression in samples compared with in control samples; negative $\log \mathrm{FC}$ values refer to downregulated expression in IDD samples compared with in control samples. DEGs, differentially expressed genes; FC, fold change; FDR, false discovery rates; IDD, intervertebral disc degeneration.

IDD. TGFB1 suppresses TNF- $\alpha$-mediated upregulation of syndecan-4 and MMP3 via ERK1/2 signaling pathways. In the present study, TGFB1 was significantly upregulated in patients with IDD compared with in control individuals. This may be representative of the inflammatory activity present in IDD, and TGFB1 expression may be increased as an inflammatory stress response to antagonize inflammation (36); this was also indirectly reflected by the lower expression of several TGFB1-associated inflammatory genes, including C-X-C motif chemokine ligand (CXCL) 1 and CXCL2 in this study. However, to the best of our knowledge, the regulatory mechanisms of TGFB1 in IDD have not been explored.

The present study revealed that hsa-miR-185-5p may regulate the expression of TGFB1, whereas MALAT1 and 

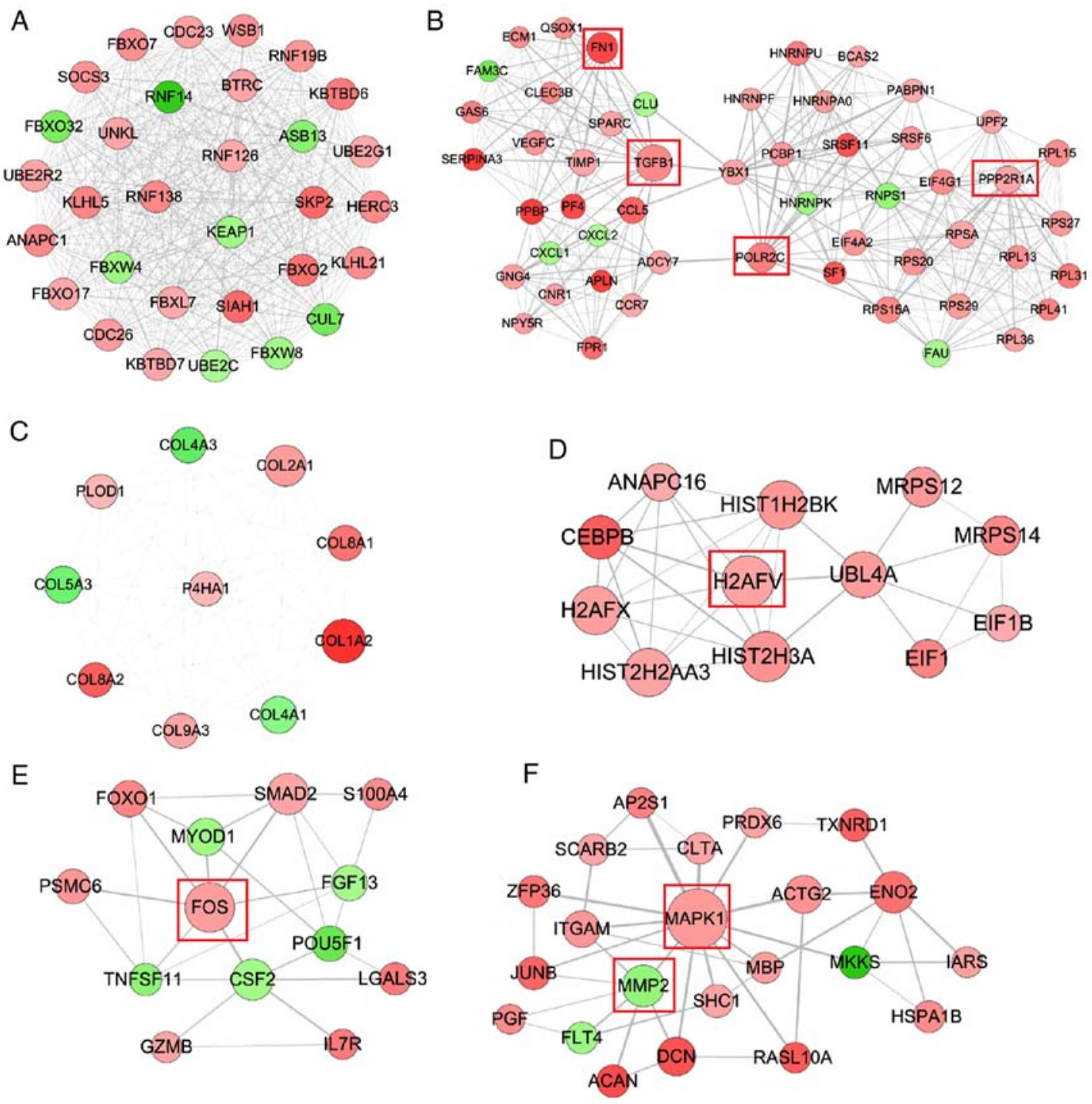

Figure 2. Modules extracted from the protein-protein interaction network. (A) Module 1; (B) module 2; (C) module 3; (D) module 4; (E) module 5; and (F) module 6. Red represents upregulated expression, whereas green represents downregulated expression. The larger the size of the node (protein), the higher its degree (number of interactions). Hub genes are indicated by red boxes.

hsa_circRNA_102348 may function as ceRNAs to interact with hsa-miR-185-5p. To the best of our knowledge, no previous studies have investigated the roles of hsa-miR-185-5p, MALAT1 and hsa_circRNA_102348 in IDD. However, their known mechanisms in regulating cell proliferation in other diseases may indirectly explain their roles in IDD. Notably, Cheng et al (37) demonstrated that miR-185 inhibits cell proliferation, while promoting apoptosis and autophagy through negative regulation of TGFB1 in nasopharyngeal carcinoma. miR-185 mimics have been observed to exert inhibitory effects on osteoblasts through downregulating the Wnt/ß-catenin axis (38). Li et al (39) demonstrated that MALAT1 promotes proliferation of cardiac progenitor cells under hypoxic conditions and alleviates myocardial infarction-induced heart failure. Furthermore, Li et al (40) revealed that MALAT1 promotes osteosarcoma cell growth and predicts an unfavorable outcome. Hereby, it was hypothesized that miR-185 may be downregulated and MALAT1 may be upregulated in IDD; this hypothesis was confirmed by the present results. Although, to the best of our knowledge, no experimental study has been conducted to validate the roles of hsa_circRNA_102348, its expression has been demonstrated to be upregulated in idiopathic pulmonary fibrosis (28); this is characterized by the abnormal deposition of ECM proteins. Therefore, the expression of hsa_circRNA_102348 may also be upregulated in IDD.

Hypoxia has been reported to promote disc cell proliferation (41). HIF1A is an important response gene under hypoxic and inflammatory conditions, which is upregulated in NP cells (42). Overexpression of HIF1A has been demonstrated to promote NP cell proliferation and lead to increased expression levels of collagen II and aggrecan in human NP cells $(8,43)$. Therefore, upregulation of HIF1A may be a protective strategy to antagonize degenerative intervertebral discs (44). In line with Wang et al (8), which reported that HIF1A was highly expressed in IDD and may exert a protective response against IDD, the mRNA expression of HIF1A was significantly upregulated in patients with IDD compared with in control individuals in the present study. However, whether HIF1A also causes the damage 
Table IV. KEGG pathway analysis of genes in modules.

A, Module 1

\begin{tabular}{|c|c|c|}
\hline Term & P-value & Genes \\
\hline hsa04120: Ubiquitin mediated proteolysis & $1.09 \times 10^{-23}$ & $\begin{array}{l}\text { ANAPC1, SOCS3, BTRC, UBE2G1, FBXO2, SKP2, CDC23, } \\
\text { HERC3, KEAP1, UBE2C, CDC26, UBE2R2, FBXW8, CUL7, } \\
\text { SIAH1 }\end{array}$ \\
\hline hsa04114: Oocyte meiosis & $1.51 \times 10^{-3}$ & ANAPC1, BTRC, CDC23, CDC26 \\
\hline hsa04110: Cell cycle & $2.19 \times 10^{-3}$ & ANAPC1, SKP2, CDC23, CDC26 \\
\hline hsa04914: Progesterone-mediated oocyte maturation & $1.48 \times 10^{-2}$ & ANAPC1, CDC23, CDC26 \\
\hline
\end{tabular}

B, Module 2

\begin{tabular}{|c|c|c|}
\hline Term & P-value & Genes \\
\hline hsa03010: Ribosome & $1.39 \times 10^{-9}$ & $\begin{array}{l}\text { RPSA, RPS27, RPS29, RPL41, RPL13, RPL31, RPL15, FAU, } \\
\text { RPL36, RPS15A, RPS20 }\end{array}$ \\
\hline hsa04062: Chemokine signaling pathway & $4.75 \times 10^{-5}$ & CXCL1, CCR7, PPBP, ADCY7, CXCL2, PF4, CCL5, GNG4 \\
\hline hsa03040: Spliceosome & $5.30 \times 10^{-3}$ & BCAS2, HNRNPK, SRSF6, PCBP1, HNRNPU \\
\hline hsa04060: Cytokine-cytokine receptor interaction & $7.15 \times 10^{-3}$ & VEGFC, CCR7, PPBP, PF4, CCL5, TGFB1 \\
\hline hsa03015: mRNA surveillance pathway & $1.24 \times 10^{-2}$ & PABPN1, PPP2R1A, UPF2, RNPS1 \\
\hline
\end{tabular}

C, Module 3

\begin{tabular}{lll}
\hline Term & P-value & Genes \\
\hline hsa04974: Protein digestion and absorption & $6.15 \times 10^{-9}$ & COL4A3, COL9A3, COL4A1, COL1A2, COL2A1, COL5A3 \\
hsa04512: ECM-receptor interaction & $7.97 \times 10^{-7}$ & COL4A3, COL4A1, COL1A2, COL2A1, COL5A3 \\
hsa05146: Amoebiasis & $1.77 \times 10^{-6}$ & COL4A3, COL4A1, COL1A2, COL2A1, COL5A3 \\
hsa04510: Focal adhesion & $2.50 \times 10^{-5}$ & COL4A3, COL4A1, COL1A2, COL2A1, COL5A3 \\
hsa04151: PI3K-Akt signaling pathway & $1.90 \times 10^{-4}$ & COL4A3, COL4A1, COL1A2, COL2A1, COL5A3 \\
hsa04611: Platelet activation & $6.93 \times 10^{-3}$ & COL1A2, COL2A1, COL5A3 \\
& & \\
\end{tabular}

D, Module 4

\begin{tabular}{|c|c|c|}
\hline Term & P-value & Genes \\
\hline hsa05322: Systemic lupus erythematosus & $1.58 \times 10^{-5}$ & HIST2H3A, HIST2H2AA3, H2AFV, HIST1H2BK, H2AFX \\
\hline hsa05034: Alcoholism & $4.74 \times 10^{-5}$ & HIST2H3A, HIST2H2AA3, H2AFV, HIST1H2BK, H2AFX \\
\hline \multicolumn{3}{|l|}{ E, Module 5} \\
\hline Term & P-value & Genes \\
\hline hsa05323: Rheumatoid arthritis & $5.45 \times 10^{-3}$ & CSF2, FOS, TNFSF11 \\
\hline hsa05200: Pathways in cancer & $1.19 \times 10^{-2}$ & FOS, FOXO1, FGF13, SMAD2 \\
\hline hsa04068: FoxO signaling pathway & $1.23 \times 10^{-2}$ & FOXO1, SMAD2, IL7R \\
\hline hsa05202: Transcriptional misregulation in cancer & $1.89 \times 10^{-2}$ & CSF2, FOXO1, GZMB \\
\hline hsa04060: Cytokine-cytokine receptor interaction & $3.40 \times 10^{-2}$ & CSF2, TNFSF11, IL7R \\
\hline hsa05166: Human T-cell leukemia virus 1 infection & $4.14 \times 10^{-2}$ & CSF2, FOS, SMAD2 \\
\hline
\end{tabular}

F, Module 6

\begin{tabular}{lll}
\hline Term & P-value & Genes \\
\hline
\end{tabular}


Table I. IV. Continued.

F, Module 6

\begin{tabular}{lll}
\hline Term & P-value & Genes \\
\hline hsa04510: Focal adhesion & $1.30 \times 10^{-2}$ & MAPK1, PGF, FLT4, SHC1 \\
hsa04015: Rap1 signaling pathway & $1.37 \times 10^{-2}$ & MAPK1, PGF, FLT4, ITGAM \\
hsa04014: Ras signaling pathway & $1.67 \times 10^{-2}$ & MAPK1, PGF, FLT4, SHC1 \\
\hline
\end{tabular}

KEGG pathways were predicted using the Database for Annotation, Visualization and Integrated Discovery online tool (version 6.8; david. abcc.ncifcrf.gov) (26). KEGG, Kyoto Encyclopedia of Genes and Genomes.

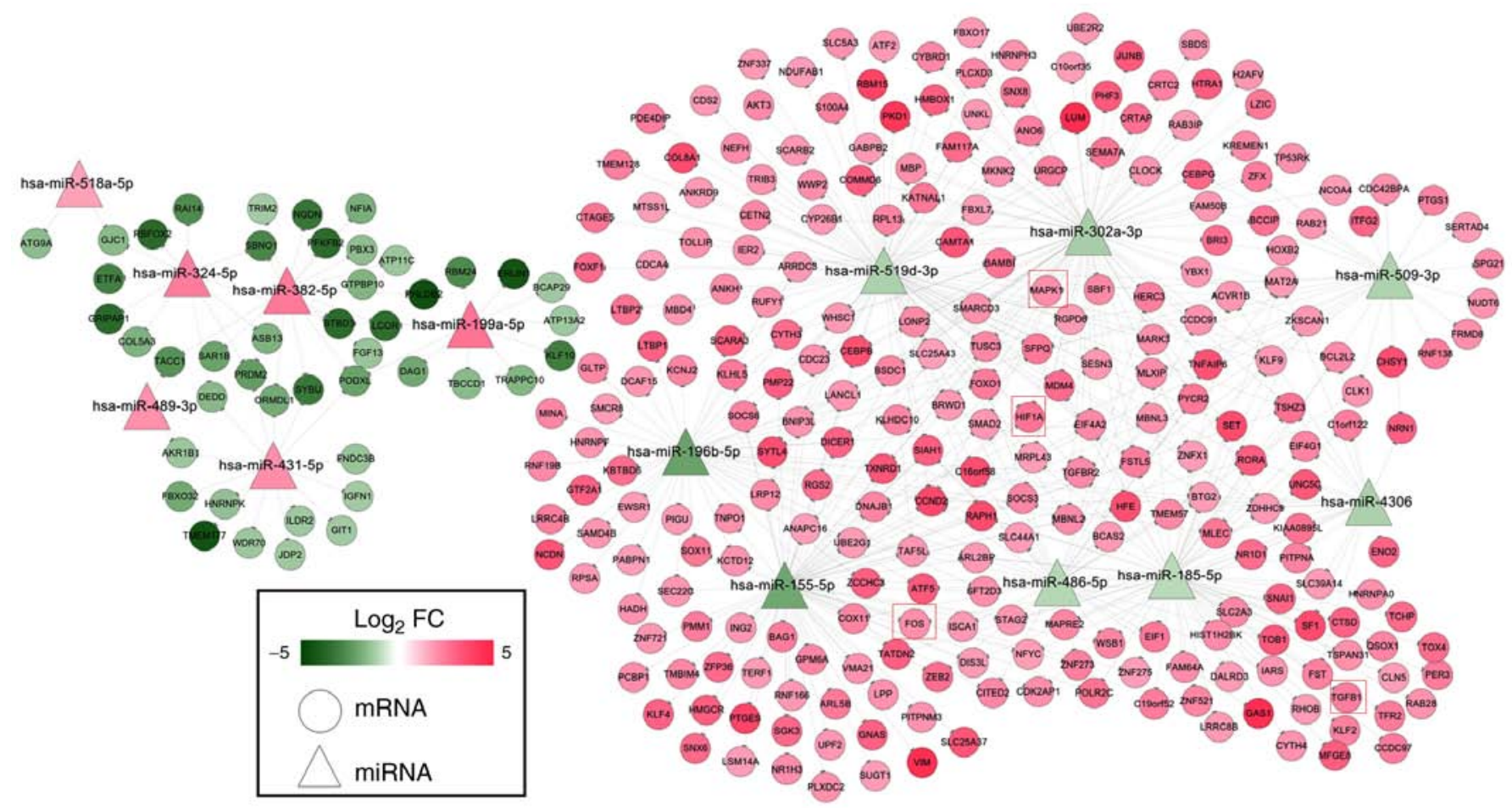

Figure 3. miRNA-mRNA interaction network. Red represents upregulated expression, whereas green represents downregulated expression. Triangular nodes represent miRNAs and oval nodes represent mRNAs. Hub genes are indicated by red boxes. FC, fold change; miRNA/miR, microRNA.

observed in IDD remains unclear and requires further investigation. Furthermore, the present study demonstrated that hsa-miR-155-5p and hsa-miR-302a-3p could regulate HIF1A expression. MALAT1 could sponge hsamiR-155-5p, whereas hsa_circRNA_102399 could sponge hsa-miR-302a-3p. Although their interaction mechanisms have not been analyzed in IDD, the roles of some miRNAs and IncRNAs have been confirmed in IDD and other diseases. For example, Wang et al (45) and Ye et al (46) demonstrated that miR-155 is downregulated in degenerative NP by microarray analysis; this was confirmed by reverse transcription-quantitative polymerase chain reaction. Zhang et al (47) demonstrated that upregulation of MALAT1 restrains IDD through suppressing inflammation and NP apoptosis, and promoting cell proliferation (47). Using gainand loss-of-function experiments, miR-155 downregulation has been revealed to be mediated by MALAT1 (48), whereas
miR-155 has also been demonstrated to directly target and silence HIF1A (49).

FOS is a member of the AP-1 transcription factor family, which has been demonstrated to be associated with numerous cellular processes, such as inhibition of chondrocyte differentiation (50), whereas chondrocyte transplantation has been proved to be a feasible and biologically relevant technique to repair disc damage and retard disc degeneration $(51,52)$. Therefore, it was hypothesized that upregulated expression of FOS may be a risk factor that promotes the development of IDD. This hypothesis has been confirmed by a recent study, which used the AP-1 selective inhibitor T-5224 to demonstrate that inhibition of c-Fos/AP-1 prevents disc degeneration and associated pain (53). As an inflammatory response gene (54), FOS was revealed to be upregulated in this study. Similar to TGFB1, hsa-miR-185-5p was shown to regulate the expression of 
Table V. KEGG pathway analysis of genes in regulatory networks.

A, miRNA-mRNA network

\begin{tabular}{lll}
\hline Term & P-value & \multicolumn{1}{c}{ Genes } \\
\hline hsa04350: TGF-beta signaling pathway & $2.02 \times 10^{-4}$ & MAPK1, ACVR1B, LTBP1, FST, TGFBR2, SMAD2, TGFB1 \\
hsa04120: Ubiquitin mediated proteolysis & $1.80 \times 10^{-3}$ & WWP2, SOCS3, UBE2G1, CDC23, SIAH1, HERC3, UBE2R2 \\
hsa04520: Adherens junction & $2.98 \times 10^{-3}$ & MAPK1, ACVR1B, TGFBR2, SMAD2, SNAI1 \\
hsa04010: MAPK signaling pathway & $5.07 \times 10^{-3}$ & MAPK1, FOS, ACVR1B, TGFBR2, MKNK2, FGF13, AKT3, \\
& & TGFB1, ATF2 \\
hsa04115: p53 signaling pathway & $8.57 \times 10^{-3}$ & CCND2, SIAH1, MDM4, SESN3 \\
hsa00051: Fructose and mannose metabolism & $9.51 \times 10^{-3}$ & PFKFB2, AKR1B1, PMM1 \\
hsa04110: Cell cycle & $1.24 \times 10^{-2}$ & CCND2, CDC23, SMAD2, TGFB1, STAG2 \\
hsa04150: mTOR signaling pathway & $1.89 \times 10^{-2}$ & MAPK1, HIF1A, AKT3 \\
hsa04620: Toll-like receptor signaling pathway & $2.02 \times 10^{-2}$ & MAPK1, FOS, TOLLIP, AKT3 \\
hsa04662: B cell receptor signaling pathway & $3.20 \times 10^{-2}$ & MAPK1, FOS, AKT3 \\
hsa04660: T cell receptor signaling pathway & $4.97 \times 10^{-2}$ & MAPK1, FOS, AKT3 \\
\hline
\end{tabular}

B, lncRNA-ceRNA network

\begin{tabular}{|c|c|c|}
\hline Term & P-value & Genes \\
\hline hsa04350: TGF-beta signaling pathway & $2.36 \times 10^{-4}$ & $\begin{array}{l}\text { ACVR1B, MAPK1, LTBP1, FST, TGFBR2, SMAD2, BAMBI, } \\
\text { TGFB1 }\end{array}$ \\
\hline hsa04068: FoxO signaling pathway & $8.21 \times 10^{-4}$ & $\begin{array}{l}\text { MAPK1, SGK3, CCND2, TGFBR2, FOXO1, SMAD2, KLF2, } \\
\text { TGFB1, AKT3 }\end{array}$ \\
\hline hsa04668: TNF signaling pathway & $2.07 \times 10^{-2}$ & MAPK1, FOS, CEBPB, SOCS3, AKT3, ATF2 \\
\hline hsa04931: Insulin resistance & $2.22 \times 10^{-2}$ & SOCS3, TRIB3, FOXO1, MLXIP, AKT3, NR1H3 \\
\hline hsa05202: Transcriptional misregulation in cancer & $3.90 \times 10^{-2}$ & CEBPB, CCND2, TGFBR2, FOXO1, WHSC1, PBX3, EWSR1 \\
\hline
\end{tabular}

C, circRNA-ceRNA network

\begin{tabular}{lll}
\hline Term & P-value & Genes \\
\hline hsa04350: TGF-beta signaling pathway & $4.18 \times 10^{-4}$ & MAPK1, ACVR1B, LTBP1, FST, TGFBR2, SMAD2, TGFB1 \\
hsa04520: Adherens junction & $1.11 \times 10^{-2}$ & MAPK1, ACVR1B, TGFBR2, SMAD2, SNAI1 \\
hsa04120: Ubiquitin mediated proteolysis & $1.93 \times 10^{-2}$ & SOCS3, UBE2G1, CDC23, SIAH1, HERC3, UBE2R2 \\
hsa04115: p53 signaling pathway & $4.19 \times 10^{-2}$ & CCND2, SIAH1, MDM4, SESN3 \\
hsa04110: Cell cycle & $4.53 \times 10^{-2}$ & CCND2, CDC23, SMAD2, TGFB1, STAG2 \\
hsa05220: Chronic myeloid leukemia & $4.53 \times 10^{-2}$ & MAPK1, ACVR1B, TGFBR2, TGFB1 \\
hsa04010: MAPK signaling pathway & $4.82 \times 10^{-2}$ & MAPK1, FOS, ACVR1B, TGFBR2, MKNK2, FGF13, TGFB1 \\
\hline
\end{tabular}

$\mathrm{D}$, Integrated ceRNA network

\begin{tabular}{lll}
\hline Term & P-value & Genes \\
\hline hsa04350: TGF-beta signaling pathway & $7.61 \times 10^{-4}$ & ACVR1B, LTBP1, FST, TGFBR2, SMAD2, TGFB1 \\
hsa04068: FoxO signaling pathway & $5.92 \times 10^{-3}$ & CCND2, TGFBR2, FOXO1, SMAD2, KLF2, TGFB1 \\
hsa05202: Transcriptional misregulation in cancer & $1.49 \times 10^{-2}$ & CCND2, TGFBR2, FOXO1, WHSC1, PBX3, EWSR1 \\
hsa04144: Endocytosis & $4.72 \times 10^{-2}$ & TGFBR2, CYTH4, RUFY1, SMAD2, CYTH3, TGFB1 \\
hsa04110: Cell cycle & $4.92 \times 10^{-2}$ & CCND2, SMAD2, TGFB1, STAG2
\end{tabular}

KEGG pathway analysis was conducted using the Database for Annotation, Visualization and Integrated Discovery online tool (version 6.8; david.abcc.ncifcrf.gov) (26). ceRNAs, competing endogenous RNAs; circRNA, circular RNA; KEGG, Kyoto Encyclopedia of Genes and Genomes; lncRNA, long noncoding RNA; miRNA, microRNA. 


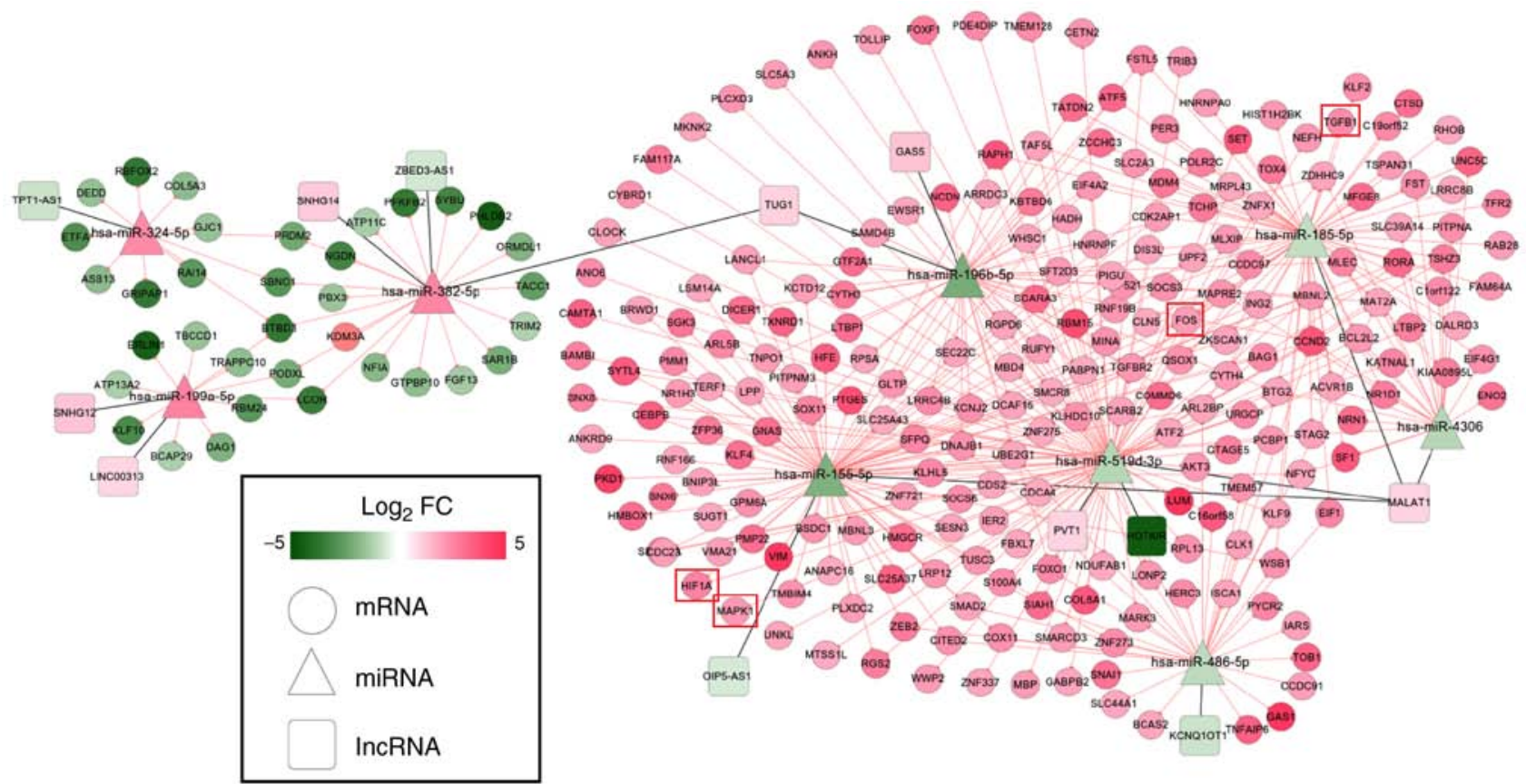

Figure 4. Competing endogenous RNA interaction network of lncRNA-miRNA-mRNA. Red represents upregulated expression, whereas green represents downregulated expression. Square nodes represent lncRNAs, triangular nodes represent miRNAs and oval nodes represent mRNAs. Hub genes are indicated by red boxes. FC, fold change; lncRNA, long noncoding RNA; miRNA/miR, microRNA.

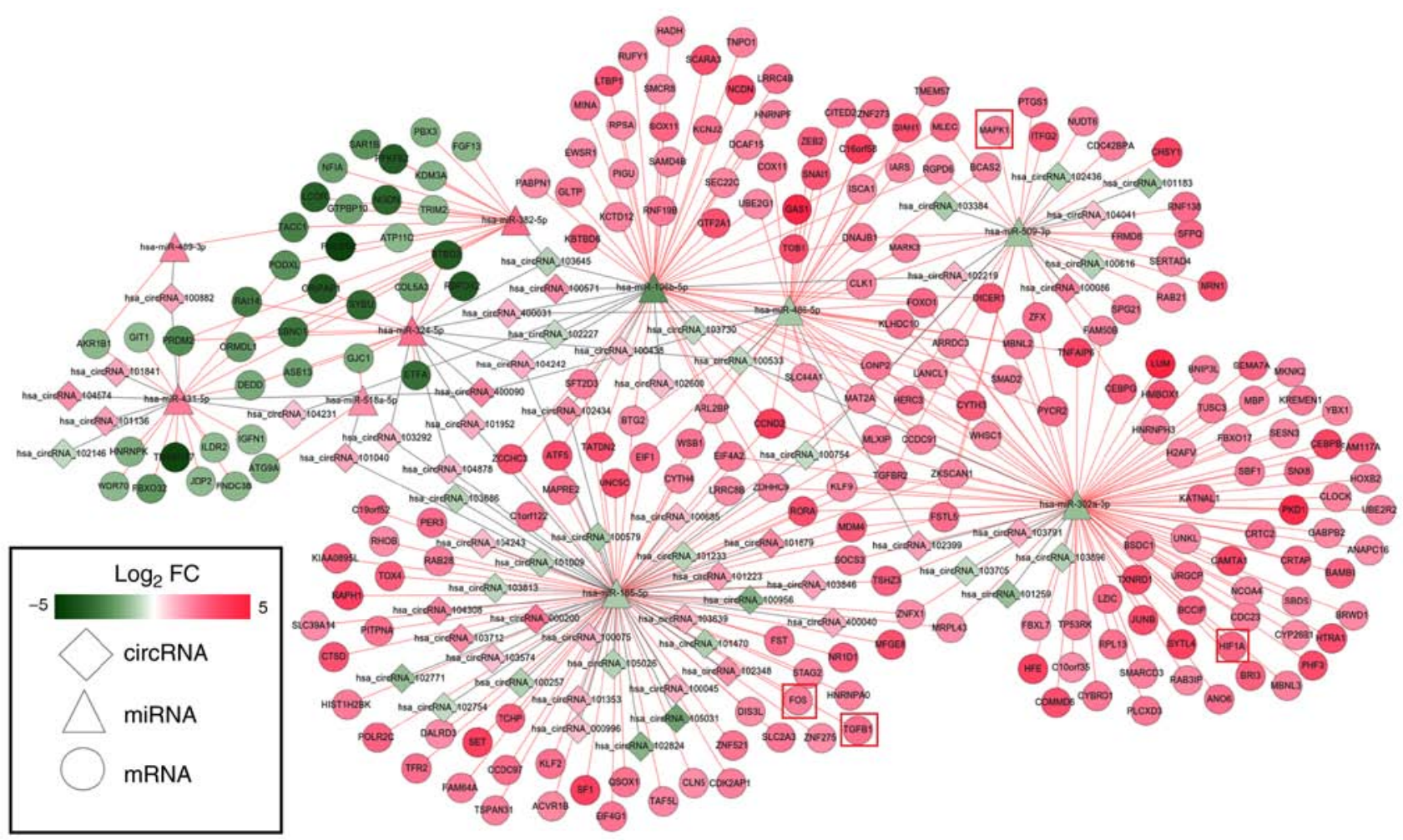

Figure 5. Competing endogenous RNA interaction network of circRNA-miRNA-mRNA. Red represents upregulated expression, whereas green represents downregulated expression. Diamond nodes represent circRNAs, triangular nodes represent miRNAs and oval nodes represent mRNAs. Hub genes are indicated by red boxes. circRNA, circular RNA; FC, fold change; miRNA/miR, microRNA.

FOS. Furthermore, MALAT1 and hsa_circRNA_102348 could sponge hsa-miR-185-5p.

Degeneration of the intervertebral disc can be mediated by several pathways. Among them, the MAPK pathways (including
JNK, ERK and p38 MAPK) have garnered extensive attention. It has been reported that blockade of p38 using Sb 202190 in NP cells can diminish the production of factors associated with inflammation, pain, and disc catabolism, and slow the course of 


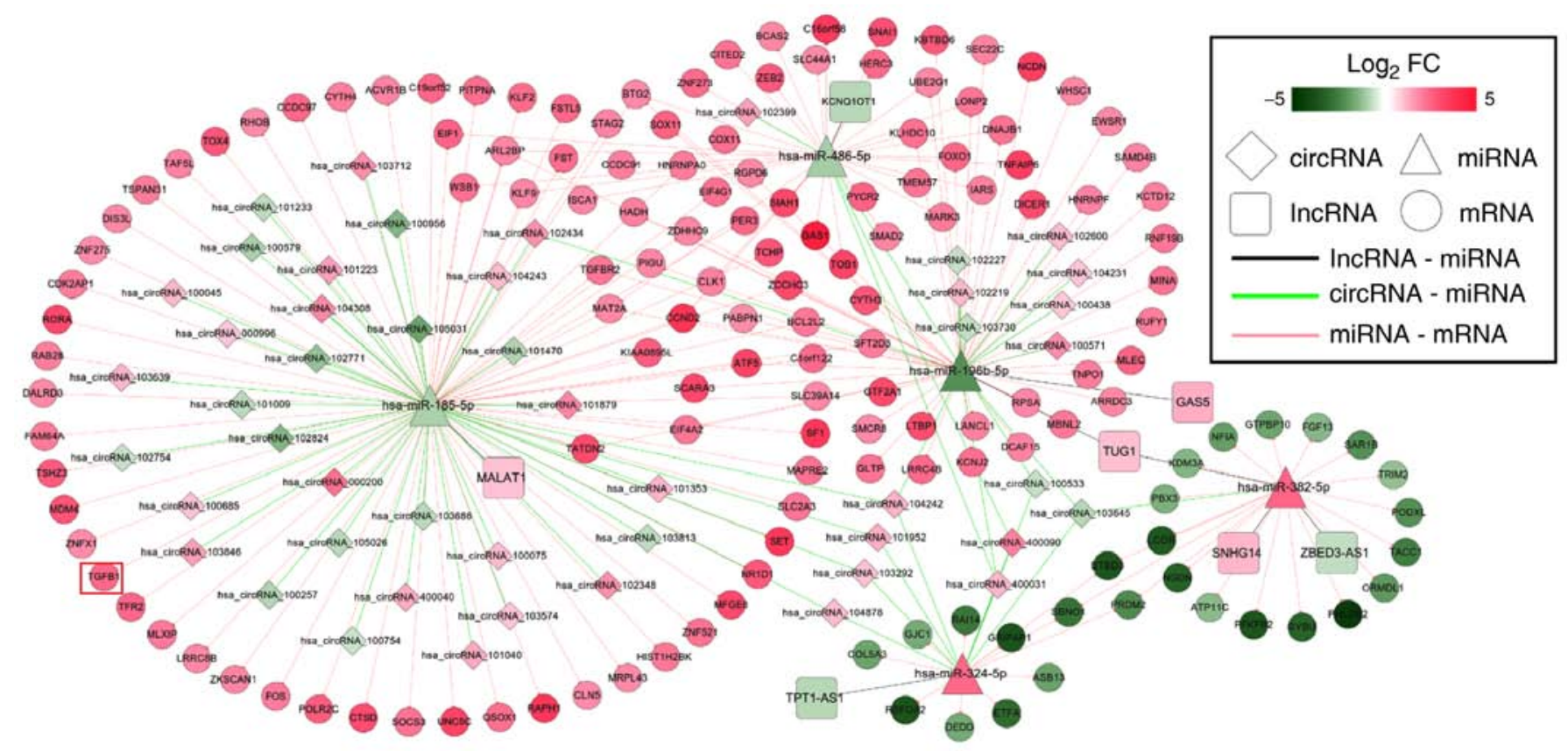

Figure 6. Integrated competing endogenous RNA interaction network of IncRNA/circRNA-miRNA-mRNA. Red represents upregulated expression, whereas green represents downregulated expression. Diamond nodes represent circRNAs, square nodes represent lncRNAs, triangular nodes represent miRNAs and oval nodes represent mRNAs. Hub genes are indicated by red boxes. circRNA, circular RNA; FC, fold change; lncRNA, long noncoding RNA; miRNA/miR, microRNA.

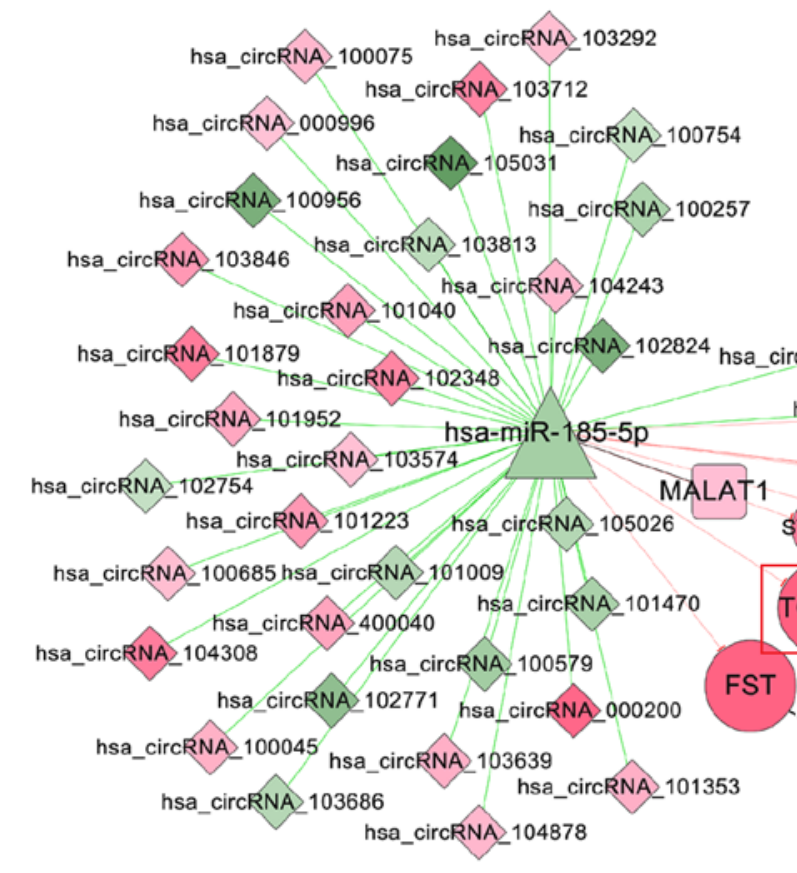


with IDD patients in this study. Furthermore, the present study demonstrated that MALAT1 could sponge hsa-miR-519d-3p to promote the expression of MAPK1, and hsa_circRNA_100086 could sponge hsa-miR-509-3p to upregulate MAPK1. The findings of the present study were indirectly confirmed by a previous study in osteosarcoma cells, in which MALAT1 was reported to promote osteosarcoma cell growth through the inhibition of miR-509, which leads to activation of the Rac1/JNK pathway (59).

There are some limitations to the present study. Firstly, the sample size was not large. An additional validation cohort should be included in further studies to analyze the expression of these identified lncRNAs, circRNAs, miRNAs and mRNAs. Secondly, this is a preliminary screening study and further experimental investigations are required to validate the interactions in the identified ceRNA axes in IDD.

In conclusion, the present study identified several lncRNA/circRNA-miRNA-mRNA interaction axes (MALAT1/hsa_circRNA_102348-hsa-miR-185-5p-TGFB1/FOS, MALAT1-hsa-miR-155-5p-HIF1A, hsa_circRNA_102399-hsamiR-302a-3p-HIF1A, MALAT1-hsa-miR-519d-3p-MAPK1 and hsa_circRNA_100086-hsa-miR-509-3p-MAPK1), which may be crucial for treatment of IDD. Clinical, in vitro and in vivo experiments will be performed in future to validate the expression patterns and interactions identified in this study in IDD.

\section{Acknowledgements}

Not applicable.

\section{Funding}

The present study was supported by the National Natural Science Foundation of China (grant no. 81601898).

\section{Availability of data and materials}

The datasets generated and/or analyzed during the present study are available in the Gene Expression Omnibus repository, https://www.ncbi.nlm.nih.gov/geo/query/acc.cgi?acc=GSE67567.

\section{Authors' contributions}

JWZ, XLZ and DJH contributed to the concept and the design of the study. WJG acquired the data. JWZ, XLZ and $\mathrm{HMH}$ conducted the statistical analysis. XDW and DJH were involved with interpretation of the data. JWZ and XLZ drafted the manuscript. DJH revised the manuscript. All authors read and approved the final manuscript.

\section{Ethics approval and consent to participate}

Not applicable.

\section{Patient consent for publication}

Not applicable.

\section{Competing interests}

The authors declare that they have no competing interests.

\section{References}

1. Gore M, Sadosky A, Stacey BR, Tai KS and Leslie D: The burden of chronic low back pain: Clinical comorbidities, treatment patterns, and health care costs in usual care settings. Spine (Phila Pa 1976) 37: E668-E677, 2012.

2. Lee CK and Langrana NA: A review of spinal fusion for degenerative disc disease: Need for alternative treatment approach of disc arthroplasty? Spine J 4 (6 Suppl): 173S-176S, 2004

3. Konovalov NA, Nazarenko AG, Asyutin DS, Zelenkov PV, Onoprienko RA, Korolishin VA, Cherkiev IU, Martynova MA, Zakirov BA, Timonin SY, et al: Modern treatments for degenerative disc diseases of the lumbosacral spine. A literature review. Zh Vopr Neirokhir Im N N Burdenko 80: 102-108, 2016 (In Russian).

4. Santosh B, Varshney A and Yadava PK: Non-coding RNAs: Biological functions and applications. Cell Biochem Funct 33: 14-22, 2015.

5. Hausser $\mathbf{J}$ and Zavolan $\mathrm{M}$ : Identification and consequences of miRNA-target interactions-beyond repression of gene expression. Nat Rev Genet 15: 599-612, 2014.

6. Salmena L, Poliseno L, Tay Y, Kats L and Pandolfi PP: A ceRNA hypothesis: The Rosetta Stone of a hidden RNA language? Cell 146: 353-358, 2011

7. Xi Y, Jiang T, Wang W, Yu J, Wang Y, Wu X and He Y: Long non-coding HCG18 promotes intervertebral disc degeneration by sponging miR-146a-5p and regulating TRAF6 expression. Sci Rep 7: 13234, 2017.

8. Wang X, Lv G, Li J, Wang B, Zhang Q and Lu C: LncRNARP11-296A18.3/miR-138/HIF1A pathway regulates the proliferation ECM synthesis of human nucleus pulposus cells (HNPCs). J Cell Biochem 118: 4862-4871, 2017.

9. Wang H, He P, Pan H, Long J, Wang J, Li Z, Liu H, Jiang W and Zheng Z: Circular RNA circ-4099 is induced by TNF- $\alpha$ and regulates ECM synthesis by blocking miR-616-5p inhibition of Sox9 in intervertebral disc degeneration. Exp Mol Med 50: 27, 2018.

10. Cheng X, Zhang L, Zhang K, Zhang G, Hu Y, Sun X, Zhao C, Li H, Li YM and Zhao J: Circular RNA VMA21 protects against intervertebral disc degeneration through targeting miR-200c and X linked inhibitor-of-apoptosis protein. Ann Rheum Dis 77: 770-779, 2018.

11. Qu Z, Quan Z, Zhang Q, Wang Z, Song Q, Zhuang X, Fu C, Xu F, Liu Y, Wang Y, et al: Comprehensive evaluation of differential lncRNA and gene expression in patients with intervertebral disc degeneration. Mol Med Rep 18: 1504-1512, 2018.

12. Lan PH, Liu ZH, Pei YJ, Wu ZG, Yu Y, Yang YF, Liu X, Che L, Ma CJ, Xie YK, et al: Landscape of RNAs in human lumbar disc degeneration. Oncotarget 7: 63166-63176, 2016.

13. Liu X, Che L, Xie YK, Hu QJ, Ma CJ, Pei YJ, Wu ZG, Liu ZH, Fan LY and Wang HQ: Noncoding RNAs in human intervertebral disc degeneration: An integrated microarray study. Genom Data 5: 80-81, 2015.

14. Wan ZY, Song F, Sun Z, Chen YF, Zhang WL, Samartzis D, Ma CJ, Che L, Liu X, Ali MA, et al: Aberrantly expressed long noncoding RNAs in human intervertebral disc degeneration: A microarray related study. Arthritis Res Ther 16: 465, 2014.

15. Ritchie ME, Phipson B, Wu D, Hu Y, Law CW, Shi W and Smyth GK: limma powers differential expression analyses for RNA-sequencing and microarray studies. Nucleic Acids Res 43: e47, 2015

16. Thissen D, Steinberg L and Kuang D: Quick and easy implementation of the benjamini-hochberg procedure for controlling the false positive rate in multiple comparisons. J Educ Behav Stat 27: 77-83, 2002.

17. Kohl M, Wiese S and Warscheid B: Cytoscape: Software for visualization and analysis of biological networks. Methods Mol Biol 696: 291-303, 2011.

18. Szklarczyk D, Franceschini A, Wyder S, Forslund K, Heller D, Huerta-Cepas J, Simonovic M, Roth A, Santos A, Tsafou KP, et al: STRING v10: Protein-protein interaction networks, integrated over the tree of life. Nucleic Acids Res 43 (Database Issue): D447-D452, 2015.

19. Tang Y, Li M, Wang J, Pan Y and Wu FX: CytoNCA: A cytoscape plugin for centrality analysis and evaluation of protein interaction networks. Biosystems 127: 67-72, 2015.

20. Bader GD and Hogue CW: An automated method for finding molecular complexes in large protein interaction networks. BMC Bioinformatics 4: 2, 2003. 
21. Li JH, Liu S, Zhou H, Qu LH and Yang JH: starBase v2.0: Decoding miRNA-ceRNA, miRNA-ncRNA and protein-RNA interaction networks from large-scale CLIP-Seq data. Nucleic Acids Res 42 (Database Issue): D92-D97, 2014.

22. Li Y, Qiu C, Tu J, Geng B, Yang J, Jiang T and Cui Q: HMDD v2.0: A database for experimentally supported human microRNA and disease associations. Nucleic Acids Res 42 (Database Issue): D1070-D1074, 2014.

23. Glažar P, Papavasileiou P and Rajewsky N: CircBase: A database for circular RNAs. RNA 20: 1666-1670, 2014.

24. Kozomara A and Griffiths-Jones S: miRBase: Annotating high confidence microRNAs using deep sequencing data. Nucleic Acids Res 42 (Database Issue): D68-D73, 2014.

25. Betel D, Koppal A, Agius P, Sander C and Leslie C: Comprehensive modeling of microRNA targets predicts functional non-conserved and non-canonical sites. Genome Biol 11: R90, 2010.

26. Huang da W, Sherman BT and Lempicki RA: Systematic and integrative analysis of large gene lists using DAVID bioinformatics resources. Nat Protoc 4: 44-57, 2009.

27. Davis AP, Grondin CJ, Johnson RJ, Sciaky D, King BL, McMorran R, Wiegers J, Wiegers TC and Mattingly CJ: The comparative toxicogenomics database: Update 2017. Nucleic Acids Res 45 (D1): D972-D978, 2017.

28. Li R, Wang Y, Song X, Sun W, Zhang J, Liu Y, Li H, Meng C, Zhang J, Zheng Q and Lv C: Potential regulatory role of circular RNA in idiopathic pulmonary fibrosis. Int J Mol Med 42: 3256-3268, 2018.

29. Liu Y, Yu T, Ma XX, Xiang HF, Hu YG and Chen BH: Lentivirus-mediated TGF- $\beta 3$, CTGF and TIMP1 gene transduction as a gene therapy for intervertebral disc degeneration in an in vivo rabbit model. Exp Ther Med 11: 1399-1404, 2016

30. Yue B, Lin Y, Ma X, Xiang H, Qiu C, Zhang J, Li L and Chen B Survivin-TGFB3-TIMP1 gene therapy via lentivirus vector slows the course of intervertebral disc degeneration in an in vivo rabbit model. Spine (Phila Pa 1976) 41: 926-934, 2016

31. Yang H, Cao C, Wu C, Yuan C, Gu Q, Shi Q and Zou J: TGF- $\beta 1$ suppresses inflammation in cell therapy for intervertebral disc degeneration. Sci Rep 5: 13254, 2015.

32. Li W, Liu T, Wu L, Chen C, Jia Z, Bai X and Ruan D: Blocking the function of inflammatory cytokines and mediators by using IL-10 and TGF- $\beta$ : A potential biological immunotherapy for intervertebral disc degeneration in a beagle model. Int J Mol Sci 15: 17270-17283, 2014.

33. Zhang J, Li Z, Chen F, Liu H, Wang H, Li X, Liu X, Wang J and Zheng Z: TGF- $\beta 1$ suppresses CCL3/4 expression through the ERK signaling pathway and inhibits intervertebral disc degeneration and inflammation-related pain in a rat model. Exp Mol Med 49: e379, 2017.

34. Yang H, Liu H, Li X, Pan H, Li Z, Wang J and Zheng Z: TNF- $\mathrm{O}$ and TGF- $\beta 1$ regulate Syndecan-4 expression in nucleus pulposus cells: Role of the mitogen-activated protein kinase and NF- $\kappa \mathrm{B}$ pathways. Connect Tissue Res 56: 281-287, 2015

35. Yang H, Gao F, Li X, Wang J, Liu H and Zheng Z: TGF- $\beta 1$ antagonizes TNF- $\alpha$ induced up-regulation of matrix metalloproteinase 3 in nucleus pulposus cells: Role of the ERK1/2 pathway. Connect Tissue Res 56: 461-468, 2015.

36. Sobajima S, Shimer AL, Chadderdon RC, Kompel JF, Kim JS Gilbertson LG and Kang JD: Quantitative analysis of gene expression in a rabbit model of intervertebral disc degeneration by real-time polymerase chain reaction. Spine J 5: 14-23, 2005.

37. Cheng JZ, Chen CJ, Wang ZG and Yu D: MicroRNA-185 inhibits cell proliferation while promoting apoptosis and autophagy through negative regulation of TGF- $\beta 1 / \mathrm{mTOR}$ axis and HOXC6 in nasopharyngeal carcinoma. Cancer Biomark 23: 107-123, 2018.

38. Yao CJ, Lv Y, Zhang CJ, Jin JX, Xu LH, Jiang J, Geng B, Li H, Xia YY and Wu M: MicroRNA-185 inhibits the growth and proliferation of osteoblasts in fracture healing by targeting PTH gene through down-regulating Wnt//-catenin axis: In an animal experiment. Biochem Biophys Res Commun 501: 55-63, 2018.

39. Li L, Wang Q, Yuan Z, Chen A, Liu Z, Wang Z and Li H: LncRNA-MALAT1 promotes CPC proliferation and migration in hypoxia by up-regulation of JMJD6 via sponging miR-125 Biochem Biophys Res Commun 499: 711-718, 2018

40. Li Q, Pan X, Wang X, Jiao X, Zheng J, Li Z and Huo Y: Long noncoding RNA MALAT1 promotes cell proliferation through suppressing miR-205 and promoting SMAD4 expression in osteosarcoma. Oncotarget 8: 106648-106660, 2017.

41. Hiyama A, Skubutyte R, Markova D, Anderson DG, Yadla S, Sakai D, Mochida J, Albert TJ, Shapiro IM and Risbud MV: Hypoxia activates the notch signaling pathway in cells of the intervertebral disc: Implications in degenerative disc disease. Arthritis Rheum 63: 1355-1364, 2011.
42. Kwon WK, Moon HJ, Kwon TH, Park YK and Kim JH: The role of hypoxia in angiogenesis and extracellular matrix regulation of intervertebral disc cells during inflammatory reactions. Neurosurgery 81: 867-875, 2017.

43. Liu Z, Li C, Meng X, Bai Y, Qi J, Wang J, Zhou Q, Zhang W and Zhang X: Hypoxia-inducible factor-l $\alpha$ mediates aggrecan and collagen $\Pi$ expression via NOTCH1 signaling in nucleus pulposus cells during intervertebral disc degeneration. Biochem Biophys Res Commun 488: 554-561, 2017.

44. Chen S, Fang XQ, Wang Q, Wang SW, Hu ZJ, Zhou ZJ, Xu WB, Wang JY, Qin A and Fan SW: PHD/HIF-1 upregulates CA12 to protect against degenerative disc disease: A human sample, in vitro and ex vivo study. Lab Invest 96: 561-569, 2016

45. Wang HQ, Yu XD, Liu ZH, Cheng X, Samartzis D, Jia LT, Wu SX, Huang J, Chen J and Luo ZJ: Deregulated miR-155 promotes fas-mediated apoptosis in human intervertebral disc degeneration by targeting FADD and caspase-3. J Pathol 225: 232-242, 2011.

46. Ye D, Dai L, Yao Y, Qin S, Han X, Wen W and Liang W: miR-155 inhibits nucleus pulposus cells' degeneration through targeting ERK 1/2. Dis Markers 2016: 6984270, 2016.

47. Zhang H, Li JD, Duan DP, She W, Wang LG and Zhang FQ: The role of lncRNA MALAT1 in intervertebral degenerative disc disease. Int J Clin Exp Patho 10: 10611-10617, 2017.

48. Cao S, Wang Y, Li J, Lv M, Niu H and Tian Y: Tumor-suppressive function of long noncoding RNA MALAT1 in glioma cells by suppressing miR-155 expression and activating FBXW7 function. Am J Cancer Res 6: 2561-2574, 2016.

49. Wu X, Li J, Yang X, Bai X, Shi J, Gao J, Li Y, Han S, Zhang Y, Han F, et al: miR-155 inhibits the formation of hypertrophic scar fibroblasts by targeting HIF-1 $\alpha$ via PI3K/AKT pathway. J Mol Histol 49: 377-387, 2018.

50. Thomas DP, Sunters A, Gentry A and Grigoriadis AE: Inhibition of chondrocyte differentiation in vitro by constitutive and inducible overexpression of the c-fos proto-oncogene. J Cell Sci 113: 439-450, 2000

51. Acosta FL Jr, Metz L, Adkisson HD, Liu J, Carruthers-Liebenberg E, Milliman C, Maloney M and Lotz JC: Porcine intervertebral disc repair using allogeneic juvenile articular chondrocytes or mesenchymal stem cells. Tissue Eng Part A 17: 3045-3055, 2011.

52. Ganey T, Libera J, Moos V, Alasevic O, Fritsch KG, Meisel HJ and Hutton WC: Disc chondrocyte transplantation in a canine model: A treatment for degenerated or damaged intervertebral disc. Spine (Phila Pa 1976) 28: 2609-2620, 2003

53. Makino H, Seki S, Yahara Y, Shiozawa S, Aikawa Y, Motomura H, Nogami M, Watanabe K, Sainoh T, Ito $\mathrm{H}$, et al: A selective inhibition of c-Fos/activator protein-1 as a potential therapeutic target for intervertebral disc degeneration and associated pain. Sci Rep 7: 16983, 2017.

54. Li JK, Nie L, Zhao YP, Zhang Y, Wang X, Wang SS, Liu Y, Zhao $\mathrm{H}$ and Cheng L: IL-17 mediates inflammatory reactions via p38/c-Fos and JNK/c-Jun activation in an AP-1-dependent manner in human nucleus pulposus cells. J Transl Med 14: 77, 2016.

55. Studer RK, Aboka AM, Gilbertson LG, Georgescu H, Sowa G, Vo N and Kang JD: p38 MAPK inhibition in nucleus pulposus cells: A potential target for treating intervertebral disc degeneration. Spine (Phila Pa 1976) 32: 2827-2833, 2007.

56. Chen B, Wang HT, Yu B, Zhang JD and Feng Y: Carthamin yellow inhibits matrix degradation and inflammation induced by LPS in the intervertebral disc via suppression of MAPK pathway activation. Exp Ther Med 4: 1614-1620, 2017.

57. Liu C, Yang H, Gao F, Li X, An Y, Wang J and Jin A: Resistin promotes intervertebral disc degeneration by upregulation of ADAMTS-5 through p38 MAPK signaling pathway. Spine (Phila Pa 1976) 41: 1414-1420, 2016

58. Yokoyama K, Hiyama A, Arai F, Nukaga T, Sakai D and Mochida J: C-Fos regulation by the MAPK and PKC pathways in intervertebral disc cells. PLoS One 8: e73210, 2013.

59. Zhang Y, Dai Q, Zeng F and Liu H: MALAT1 promotes the proliferation and metastasis of osteosarcoma cells by activating the Rac1/JNK pathway via targeting MiR-509. Oncol Res, Apr 27, 2018 (Epub ahead of print).

This work is licensed under a Creative Commons Attribution-NonCommercial-NoDerivatives 4.0 International (CC BY-NC-ND 4.0) License. 